\title{
How to Save a Limb: Identification of Pyoderma Gangrenosum
}

\author{
Emily K. Haque, BSA; Raghavendra Girijala, MD; Clifton Molak, MD
}

\section{PRACTICE POINTS}

- Pyoderma gangrenosum (PG) frequently is misdiagnosed due to its similar presentation to other skin and soft tissue infections (SSTIs). Patients with known risk factors for PG should be evaluated with a high index of suspicion to ensure early diagnosis and avoid serious complications. Common associations include inflammatory bowel disease (IBD), hematologic malignancies, and rheumatologic disorders.

- Response to treatment may be used to guide management when the diagnosis of SSTIs vs PG cannot be distinguished with clinical and histologic findings alone. In a worsening ulcer that has failed antibiotic therapy, clinicians should consider the diagnosis of $P G$ and the risk of pathergy prior to surgical intervention such as debridement.

- Although typically a diagnosis of exclusion, clinicians can consider the use of diagnostic criteria for PG in patients of high clinical suspicion. A trial of immunosuppressants can be considered after infection has been ruled out.

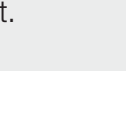

We report the case of a 67-year-old woman with a painful expanding ulcer on the left leg of 2 months' duration that initially was diagnosed as a skin and soft tissue infection (SSTI) with plans for surgical debridement. Upon dermatologic consultation, surgery was canceled due to the possible diagnosis of pyoderma gangrenosum (PG) and risk of pathergy. Notable features of this patient included a history of inflammatory bowel disease (IBD), poor response to antibiotics, chronicity, lack of signs of sepsis, potential complications of surgical intervention, and ultimately a response to immunosuppressive agents.

Cutis. 2021;107:328-331.

\section{Case Report}

A 67-year-old woman presented with a painful expanding ulcer on the left leg and a new nearby ulcer of 2 months' duration. She initially was seen 2 months prior for a wound on the left knee due to a fall as well as cellulitis, which was treated with intravenous vancomycin and ceftriaxone. Wound cultures were negative for bacteria, and she was discharged without antibiotics. She presented to the emergency department 1 month later for malodorous discharge of the first ulcer with zero systemic inflammatory response syndrome criteria; no fever; and no abnormal heart rate, respiratory rate, or leukocyte count. She was discharged with wound care. After 3 weeks, she returned with a second ulcer and worsening drainage but zero systemic inflammatory response syndrome criteria. She had a medical history of Crohn disease with 9-year remission, atrial fibrillation, pacemaker, mitral valve replacement, chronic obstructive pulmonary disease, and a 51 pack-year smoking history.

Physical examination of the left leg revealed a $3 \times 3-\mathrm{cm}$ deep lesion (ulcer A) on the distal left thigh located superomedial to the knee (Figure 1) as well as a $2 \times 1$-cm deep lesion (ulcer B) on the anteromedial knee with undermining and tunneling (Figure 2). A large amount of malodorous tan bloody discharge was present on both ulcers. There were no signs of induration or crepitus.

Due to concerns of skin and soft tissue infection (SSTI) or osteomyelitis, a bone scan and wound and blood cultures were ordered. The patient was started on vancomycin and piperacillin-tazobactam in the emergency department, which later was augmented with cefepime. Trauma surgery scheduled debridement for the following morning with suspicion of necrotizing

From Baylor University Medical Center, Department of Internal Medicine, Dallas, Texas. Ms. Haque also is from Texas A\&M College of Medicine, Bryan. The authors report no conflict of interest.

Correspondence: Emily K. Haque, BSA, 3500 Gaston Ave, 6-Roberts, Dallas, TX 75246 (Emily.Haque@tamu.edu).

doi: $10.12788 /$ cutis.0267 


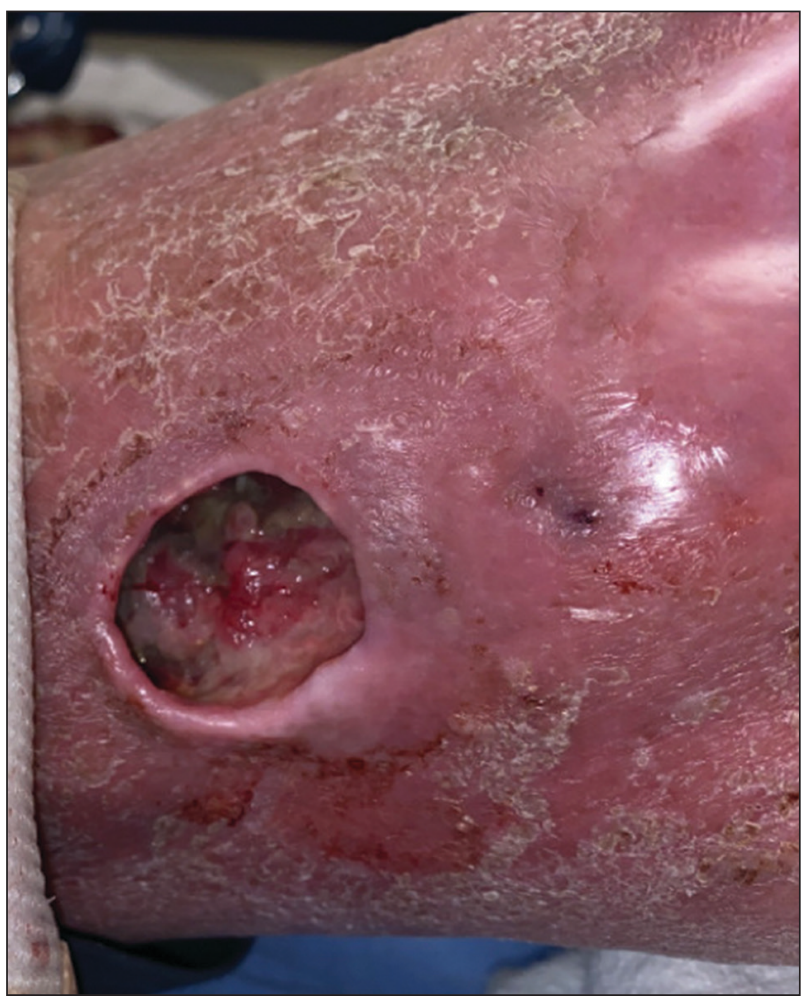

FIGURE 1. Primary distal medial thigh ulcer demonstrating a violaceous ulcer edge (ulcer A).

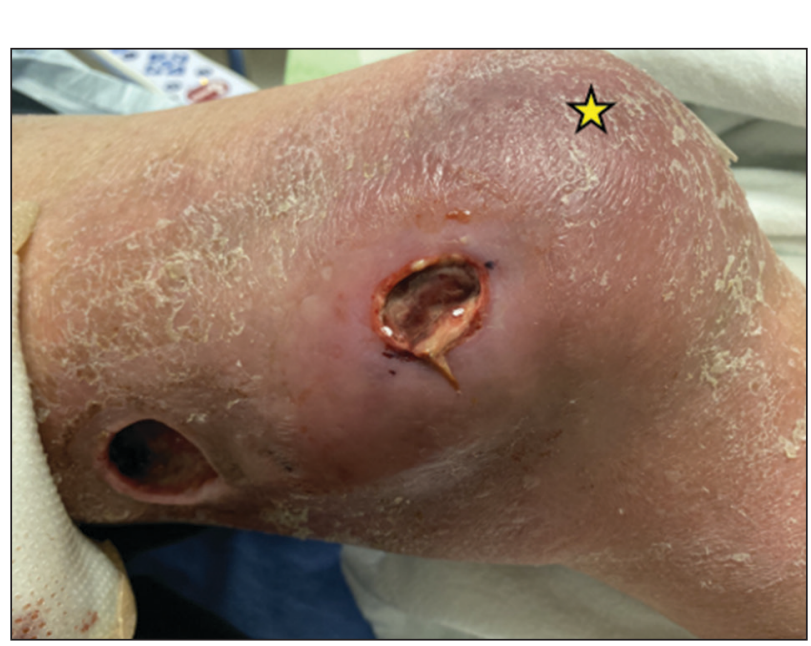

FIGURE 2. Secondary ulcer located anteromedial to the patella (yellow star) demonstrating undermining and tunneling (ulcer B).

fasciitis. Additional consultations were requested, including infectious disease, wound care, and dermatology. Dermatology evaluated the wound, performed a punch biopsy, and canceled debridement due to unclear diagnosis. The clinical differential at that time included pyoderma gangrenosum (PG), atypical vasculitis, or infection. Additional workup revealed positive antineutrophil cytoplasmic antibodies but negative proteinase 3 and myeloperoxidase, disfavoring

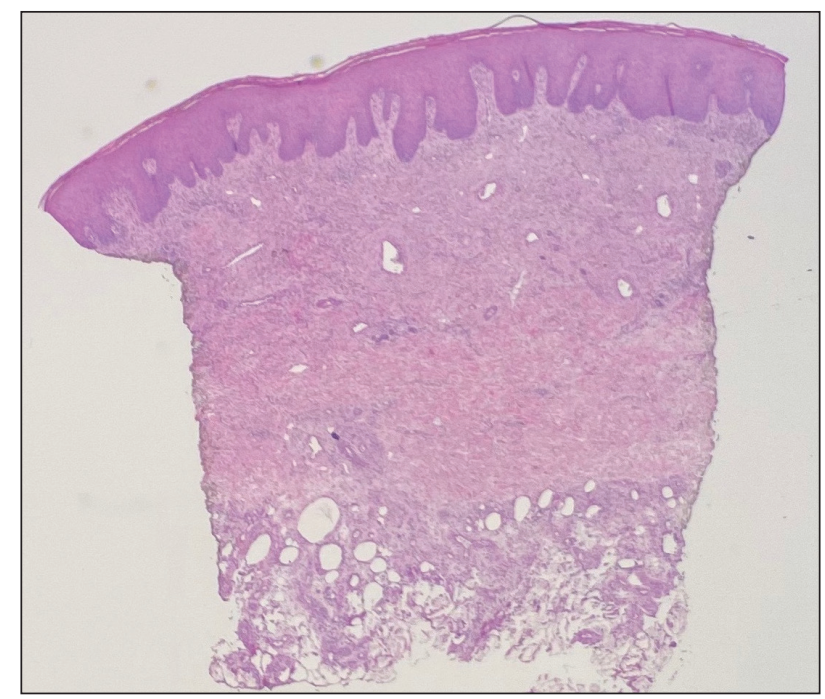

FIGURE 3. Punch biopsy of the primary ulcer showed subcutaneous and dermal necrosis $(\mathrm{H} \& \mathrm{E}$, original magnification $\times 2)$.

vasculitis. Wound cultures grew Staphylococcus aureus and Pseudomonas aeruginosa.

Histologic evaluation revealed deep dermal necrosis with a mixed inflammatory infiltrate (Figure 3) and no organisms or vasculitis. Antibiotics were discontinued, and she was discharged on a 14-day course of prednisone $60 \mathrm{mg}$ daily for empirical treatment of PG with dermatology follow-up. Medical management included a 6-month course of dapsone that was extended to 7 months because of an intensive care unit stay for a cerebrovascular accident. Daily dosing was as follows: $100 \mathrm{mg}$ for 5 months, $50 \mathrm{mg}$ for 1 month, and $25 \mathrm{mg}$ for 1 month, then stopped. She was followed with serial complete blood cell count every 1 to 2 months and home-health wound care. One month after dapsone initiation, the ulcers decreased in size. Ulcer B was fully healed after 4 months, and ulcer A was nearly closed at 6 months without any new flares.

\section{Comment}

Pyoderma gangrenosum is a rare inflammatory skin condition that classically presents as tender papules or pustules evolving into painful ulcers, most commonly on the lower extremities. Pyoderma gangrenosum has a propensity to exhibit pathergy, the hyperreactivity of the skin in response to minor trauma. This phenomenon in PG manifests as the rapid evolution from pustule to ulceration with violaceous undermining borders.

Diagnosis of PG-Pyoderma gangrenosum has been described as a diagnosis of exclusion, as its findings frequently mimic SSTIs. Important findings to obtain are histology, history, ulcer morphology, and response to treatment.

In 2018, Maverakis et $\mathrm{al}^{1}$ proposed diagnostic criteria for classic ulcerative PG (Table 1). A diagnosis of PG can be made if the patient meets 1 major criterion and 4 minor criteria. Our case met 0 major criteria and 5 minor criteria: history of inflammatory bowel disease (IBD); 
history of pustule ulcerating within 4 days of appearing; peripheral erythema, undermining border, and tenderness at ulceration site; multiple ulcerations, with at least 1 on an anterior lower leg; and decreased ulcer size within 1 month of initiating immunosuppressive medication(s). Although our patient's biopsy demonstrated a mixed infiltrate, PG was not excluded due to spontaneous resolution at the time of biopsy, emphasizing the need to biopsy subsequent new lesions if neutrophils are not initially seen. ${ }^{1}$ Pyoderma gangrenosum frequently is associated with IBD, most often Crohn disease, as seen in our patient. ${ }^{2-4}$ Although IBD classically is associated with smoking, studies have yet to conclude if smoking is a predictive factor of PG. ${ }^{5}$ Our patient presented with an initial ulcer that evolved into 2 ulcers, similar to a case of bilateral ulcers. ${ }^{6}$

Differential Diagnosis of $P G$ - Other possible diagnoses to consider are SSTI and vasculitis, the latter being disfavored by no evidence of vasculitis on biopsy and negative titers for proteinase 3 and myeloperoxidase antibodies. However, the presence of either, similar to a mixed infiltrate, does not exclude a diagnosis of PG, as they can occur simultaneously. Consequently, superinfection of a chronically open wound can occur due to underlying

\section{TABLE 1. Diagnostic Criteria for Classic Ulcerative Pyoderma Gangrenosum}

\section{Major criterion}

Biopsy of ulcer edge demonstrating neutrophilic infiltrate

\section{Minor criteria}

Exclusion of infection

Pathergy
History of IBD or inflammatory arthritis
History of papule, pustule, or vesicle ulcerating within 4 days of appearing

Peripheral erythema, undermining border, and tenderness at ulceration site

Multiple ulcerations, at least 1 on an anterior lower leg

Cribriform or "wrinkled paper" scar(s) at healed ulcer sites

Decreased ulcer size within 1 month of initiating immunosuppressive medication(s)

Abbreviations: IBD, inflammatory bowel disease; PG, pyoderma gangrenosum.

${ }^{a} \mathrm{~A}$ diagnosis of $\mathrm{PG}$ can be made if the patient meets 1 major criterion and 4 minor criteria.

TABLE 2. Pyoderma Gangrenosum vs Skin and Soft Tissue Infection

\begin{tabular}{lll}
\hline Feature & PG & SSTI \\
\hline Blood and tissue cultures & Negative $^{a}$ & Positive \\
\hline Progression & Slow & Rapid \\
\hline Morphology & Violaceous ulcer edge & Variable; red, purulent, pruritic \\
\hline Surgical intervention & Worsens wound & Improves wound \\
\hline Antibiotic therapy & No response & Improves wound \\
\hline Immunosuppressive therapy & Improves wound & Worsens wound \\
\hline
\end{tabular}

Abbreviations: PG, pyoderma gangrenosum; SSTI, skin and soft tissue infection.

${ }^{a}$ Can be positive if superinfection develops in an open wound.

bIn certain indications. 
PG. ${ }^{7}$ The differences between PG and SSTI are listed in Table 2.

Although we know PG involves neutrophilic dysfunction, the pathophysiology remains poorly understood, contributing to the lack of clinical guidelines. ${ }^{8}$ Therefore, the diagnosis of PG often is delayed and is associated with severe consequences such as necrotizing fasciitis, osteomyelitis, cosmetic morbidity, and limb amputation. ${ }^{9,10}$ Dermatologic consultation can aid in early diagnosis and avoid amputation. ${ }^{7,10}$ Amputation has been used as a last resort to preserve optimal outcomes in patients with severe PG. ${ }^{11}$

Management of $P G-A$ gold standard of treatment of PG does not exist, but the goal is to promote wound healing. Patients with limited disease typically can be managed with wound care and topical steroids or calcineurin inhibitors, though data on efficacy are limited. However, our patient had more extensive disease and needed to be treated with systemic therapy. First-line therapy for extensive disease includes oral prednisone or cyclosporine for patients who cannot tolerate systemic corticosteroids. ${ }^{12}$ Second-line and adjunctive therapy options include dapsone, minocycline, methotrexate, and infliximab. Our patient was prescribed a 7-month course of dapsone with outpatient dermatology and demonstrated resolution of both ulcers. Dapsone was tapered from a daily dose of $100 \mathrm{mg}$ to $50 \mathrm{mg}$ to $25 \mathrm{mg}$ to none over the course of 2 to 3 months. Close monitoring with wound care is recommended, and petroleum jelly can be used for dry skin around the lesion for comfort.

\section{Conclusion}

The diagnosis of PG is challenging because it relies heavily on clinical signs and often mimics SSTI. Gathering a detailed medical history is critical to make the diagnosis of PG. In a patient with associated features of PG, dermatologic consultation and biopsy of skin lesions should be considered. Physicians should evaluate for suspected PG prior to proceeding with surgical intervention to avoid unnecessary amputation. The diagnostic criteria for classic ulcerative PG are gaining wider acceptance and are a useful tool for clinicians.

\section{REFERENCES}

1. Maverakis E, Ma C, Shinkai K, et al. Diagnostic criteria of ulcerative pyoderma gangrenosum: a Delphi consensus of international experts. JAMA Dermatol. 2018;154:461-466.

2. Bisarya K, Azzopardi S, Lye G, et al. Necrotizing fasciitis versus pyoderma gangrenosum: securing the correct diagnosis! a case report and literature review. Eplasty. 2011;11:E24.

3. Perricone G, Vangeli M. Pyoderma gangrenosum in ulcerative colitis. N Engl J Med. 2018;379:E7.

4. Ashchyan HJ, Butler DC, Nelson CA, et al. The association of age with clinical presentation and comorbidities of pyoderma gangrenosum. JAMA Dermatol. 2018;154:409-413.

5. Ampuero J, Rojas-Feria M, Castro-Fernández M, et al. Predictive factors for erythema nodosum and pyoderma gangrenosum in inflammatory bowel disease. J Gastroenterol Hepatol. 2014;29:291-295.

6. Ebner DW, Hu M, Poterucha TH. 29-year-old woman with fever and bilateral lower extremity lesions. Mayo Clin Proc. 2018; 93:1659-1663.

7. Marzak H, Von Hunolstein JJ, Lipsker D, et al. Management of a superinfected pyoderma gangrenosum after pacemaker implant. HeartRhythm Case Rep. 2018;5:63-65.

8. Braswell SF, Kostopoulos TC, Ortega-Loayza AG. Pathophysiology of pyoderma gangrenosum (PG): an updated review. J Am Acad Dermatol. 2015;73:691-698.

9. Saffie MG, Shroff A. A case of pyoderma gangrenosum misdiagnosed as necrotizing infection: a potential diagnostic catastrophe. Case Rep Infect Dis. 2018;2018:8907542.

10. Haag CK, Nutan F, Cyrus JW, et al. Pyoderma gangrenosum misdiagnosis resulting in amputation: a review. J Trauma Acute Care Surg. 2019;86:307-313.

11. Sanchez IM, Lowenstein S, Johnson KA, et al. Clinical features of neutrophilic dermatosis variants resembling necrotizing fasciitis. JAMA Dermatol. 2019;155:79-84.

12. Alavi A, French LE, Davis MD, et al. Pyoderma gangrenosum: an update on pathophysiology, diagnosis and treatment. Am J Clin Dermatol. 2017; 18:355-372 